\title{
Loss of the Spectraplakin Short Stop Activates the DLK Injury Response Pathway in Drosophila
}

\author{
Vera Valakh, ${ }^{1}$ Lauren J. Walker, ${ }^{1}$ James B. Skeath, ${ }^{2}$ and Aaron DiAntonio ${ }^{1}$ \\ ${ }^{1}$ Department of Developmental Biology, Hope Center for Neurological Disorders, and ${ }^{2}$ Department of Genetics, Washington University School of Medicine, \\ St. Louis, Missouri 63110
}

The MAPKKK dual leucine zipper-containing kinase (DLK, Wallenda in Drosophila) is an evolutionarily conserved component of the axonal injury response pathway. After nerve injury, DLK promotes degeneration of distal axons and regeneration of proximal axons. This dual role in coordinating degeneration and regeneration suggests that DLK may be a sensor of axon injury, and so understanding how DLK is activated is important. Two mechanisms are known to activate DLK. First, increasing the levels of DLK via overexpression or loss of the PHR ubiquitin ligases that target DLK activate DLK signaling. Second, in Caenorhabditis elegans, a calcium-dependent mechanism, can activate DLK. Here we describe a new mechanism that activates DLK in Drosophila: loss of the spectraplakin short stop (shot). In a genetic screen for mutants with defective neuromuscular junction development, we identify a hypomorphic allele of shot that displays synaptic terminal overgrowth and a precocious regenerative response to nerve injury. We demonstrate that both phenotypes are the result of overactivation of the DLK signaling pathway. We further show that, unlike mutations in the PHR ligase Highwire, loss of function of shot activates DLK without a concomitant increase in the levels of DLK. As a spectraplakin, Shot binds to both actin and microtubules and promotes cytoskeletal stability. The DLK pathway is also activated by downregulation of the TCP1 chaperonin complex, whose normal function is to promote cytoskeletal stability. These findings support the model that DLK is activated by cytoskeletal instability, which is a shared feature of both spectraplakin mutants and injured axons.

\section{Introduction}

A conserved signaling pathway featuring the PHR ubiquitin ligase and its target, the MAPKKK DLK, regulates both neural circuit development and the axon injury response pathway (Tian and $\mathrm{Wu}, 2013)$. In Drosophila, loss of the PHR ligase Highwire leads to a dramatic expansion of the neuromuscular junction (NMJ) (Wan et al., 2000; DiAntonio et al., 2001) and promotes both proximal axon regeneration and distal axon degeneration in response to nerve injury (Xiong et al., 2010, 2012). The PHR ligase is conserved in worms and mice, where it also regulates circuit development (Schaefer et al., 2000; Zhen et al., 2000; Bloom et al., 2007; Lewcock et al., 2007; Culican et al., 2009) and the response to nerve injury (Nix et al., 2011; Babetto et al., 2013). The Highwire ligase targets the MAPKKK Wallenda/DLK, and in highwire mutants the levels of Wallenda/DLK rise and the downstream JNK/Fos pathway is overactivated (Collins et al., 2006). A

\footnotetext{
Received May 23, 2013; revised 0ct. 2, 2013; accepted Oct. 7, 2013.

Author contributions: V.V. and A.D. designed research; V.V. and L.J.W. performed research; J.B.S. contributed unpublished reagents/analytic tools; V.V. analyzed data; V.V. and A.D. wrote the paper.

This work was supported by National Institutes of Health Grants DA020812 and NS065053 to A.D. and Grant NS036570 to J.B.S. We thank members of the A.D. laboratory for helpful discussions and especially Sylvia Johnson for the help with the initial screening; Dr. Andreas Prokop for providing the rescue constructs; the Bloomington Stock Center (Indiana University) and the Vienna Drosophila RNAi Center for providing additional stocks; and the Developmental Studies Hybridoma Bank (University of lowa) for antibodies.

The authors declare no competing financial interests.

Correspondence should be addressed to Dr. Aaron DiAntonio, Department of Developmental Biology, Washington University School of Medicine, Campus Box 8103, 660 S. Euclid, St. Louis, M0 63110. E-mail: diantonio@wustl.edu.

DOI:10.1523/JNEUROSCI.2196-13.2013

Copyright $\odot 2013$ the authors $\quad 0270-6474 / 13 / 3317863-11 \$ 15.00 / 0$
}

series of recent studies demonstrate that DLK is a central component of an evolutionarily conserved axon injury response pathway, regulating both axon regeneration (Hammarlund et al., 2009; Yan et al., 2009; Xiong et al., 2010; Shin et al., 2012) and axon degeneration (Miller et al., 2009; Xiong and Collins, 2012). Despite the clear importance of DLK after nerve injury, our understanding of how DLK/Wallenda is activated in vivo is limited.

To date, two methods for activating DLK have been described. First, DLK is activated when its levels rise because of transgenic overexpression, loss of the PHR ligase, or JNK activation (Nakata et al., 2005; Collins et al., 2006; Huntwork-Rodriguez et al., 2013). Second, in Caenorhabditis elegans, an elegant $\mathrm{Ca}^{2+}$ dependent mechanism activates the DLK ortholog DLK-1 (Yan and Jin, 2012). Mammalian and Drosophila DLK, however, lack the hexapeptide sequence that mediates this form of regulation, suggesting that additional DLK activation mechanisms exist.

In this study, we demonstrate that the Drosophila spectraplakin short stop (shot) inhibits the activation of Wallenda/DLK. Spectraplakins are large multidomain proteins that cross-link actin and microtubules to regulate cytoskeletal dynamics (Suozzi et al., 2012). Previously described shot alleles are embryonic lethal, have disrupted microtubule dynamics, and exhibit severe defects in axon outgrowth (Lee and Kolodziej, 2002; Bottenberg et al., 2009; Alves-Silva et al., 2012). Here we identify a viable loss-offunction allele of shot that displays dramatic synaptic terminal overgrowth at the NMJ. In this mutant, the Wallenda/DLK pathway is overactivated leading to both NMJ overgrowth and enhanced response to axonal injury, yet unlike in highwire mutants, the levels of Wallenda/DLK are not elevated. These observations 
suggest a model in which tonic cytoskeletal destabilization in the shot mutant activates Wallenda/DLK without altering its levels. In support of this model, mutations in the TCP1 chaperonin complex, which is also required for stability of the tubulin and actin cytoskeleton (Ursic et al., 1994; Grantham et al., 2006), also activate DLK signaling. These findings led us to speculate that axonal injury activates DLK via acute cytoskeletal destabilization.

\section{Materials and Methods}

Fly stocks. Flies were maintained at $25^{\circ} \mathrm{C}$ on standard fly food. We used the following fly stocks in our studies: Canton S (WT), elav-Gal4 (Yao and White, 1994), 24B-Gal4 (Brand and Perrimon, 1993), BG380-Gal4 (Sanyal, 2009), m12-Gal4 (P(GAL4)5053A) (Klapper, 2000), $w_{n d}^{2}$ and $w n d^{3}$ (Collins et al., 2006), UAS-bsk $k^{D N}$ (Weber et al., 2000), UAS$f o s^{D N}(\mathrm{Fbz})$ (Eresh et al., 1997), and puc-LacZ (Martín-Blanco et al., 1998). The following fly lines were obtained from the Bloomington Stock Center: $\operatorname{shot}^{3}$ (Kolodziej et al., 1995), the deficiency line Df(2R)BSC383, shot RNAi line P\{TRiP.GL01286\}attP2, TCP1 $\alpha$ and TCP1 $\beta$ RNAi lines, shot rescue constructs UAS-shot $L(A)-G F P$ (shot-FL in this paper), UASshot $L(C)$-GFP (shot- $\triangle$ Calponin in this paper), UAS-shot L(A)-DeltaEFhand-GFP (shot- $\triangle E F), U A S$-shot L(A)-DeltaPlakin-GFP (Shot- $\Delta$ Plakin), UAS-shot L(A)-Deltarod1-GFP (shot- $\Delta$ Rod) (Bottenberg et al., 2009), UAS-EB1-GFP, and ppk-Gal4. UAS-shot L(A)-DeltaGAS2-GFP (shot$\triangle$ Gas2) was obtained from Andreas Prokop. UAS-wnd RNAi and RNAis against neuroligin and CG18278 were obtained from Vienna RNAi stock center (Dietzl et al., 2007).

Immunohistochemistry. Third-instar larvae were dissected in PBS and fixed in either Bouin's fixative for $5 \mathrm{~min}$ or $4 \%$ formaldehyde for $20 \mathrm{~min}$ on ice. Larvae were washed with PBS containing $0.1 \%$ Triton X-100 (PBT) and blocked in 5\% NGS in PBT for $30 \mathrm{~min}$, followed by overnight incubation in primary antibodies in 5\% NGS in PBT, three washes in PBT, incubation in secondary antibodies in 5\% NGS in PBT for $45 \mathrm{~min}$, three final washes in PBT, and equilibration in 70\% glycerol in PBS. Samples were mounted in VectaShield (Vector). The following primary antibodies were used: mouse $\alpha$-Brp, 1:500 (Developmental Studies Hybridoma Bank), mouse $\alpha$-Dlg monoclonal antibody (mAb) 4f3, 1:2000 (Developmental Studies Hybridoma Bank), rabbit $\alpha$-DGluRIII, 1:2500 (Marrus et al., 2004), rabbit $\alpha$-DVGLUT, 1:10,000 (Daniels et al., 2004), rat anti-Elav, 1:50 (7E8A10; Developmental Studies Hybridoma Bank), mouse anti-LacZ, 1:100 (40-1a; Developmental Studies Hybridoma Bank), rabbit $\alpha$-Wallenda (Collins et al., 2006), and A488 rabbit antiGFP, 1:1000 (Invitrogen). Goat Cy5-, Cy3-, and FITC-conjugated secondary antibodies against mouse and rabbit IgG were used at 1:1000 and were obtained from Jackson ImmunoResearch Laboratories. Antibodies obtained from the Developmental Studies Hybridoma Bank were developed under the auspices of the National Institute of Child Health and Human Development and maintained by the Department of Biological Sciences of the University of Iowa, Iowa City, Iowa.

Western blot. Third-instar larval brains were homogenized in ice-cold homogenization buffer ( $67 \mathrm{~mm}$ Tris- $\mathrm{HCl}$, $\mathrm{pH} 8.0,67 \mathrm{~mm} \mathrm{NaCl}, 2 \mathrm{~m}$ urea, $1 \mathrm{~mm}$ EDTA, and $1.3 \%$ SDS), and samples were run on 15\% SDS-PAGE gels according to standard procedures (Wu et al., 2005). Rabbit $\alpha$-Wallenda was used at 1:100. Mouse $\alpha$-Armadillo (Developmental Studies Hybridoma Bank) was used at 1:20. HRP-conjugated goat $\alpha$-rabbit and $\alpha$-mouse (Jackson ImmunoResearch Laboratories) were used at 1:10,000.

Imaging and analysis. Samples were imaged using a Nikon C1 confocal microscope using $40 \times$ (for VNC images) or $60 \times$ (for NMJs) objectives. All genotypes for an individual experiment were imaged at the same gain and set such that signals from the brightest genotype for a given experiment were not saturating. Both male and female larvae were used for each experiment. To quantify the mean intensity of puc-lacZ expression, the nuclei along the dorsal midline of the ventral nerve cords were selected by the staining for Elav. puc-lacZ is localized to the nuclei in those cells because of the NLS sequence fusion. The nuclei from at least 9 animals per genotype were measured and then normalized to control. To quantify Wallenda intensity in the neuropil of larval VNCs, the region was selected based on the BRP staining. At least eight larvae were used per genotype and the staining intensity was normalized to WT. Statistical analysis was performed and graphs were generated using Origin 7.0 (Origin Laboratory). ANOVA was used for comparison of samples within an experimental group. All histograms and measurements are shown as mean \pm SEM.

Live imaging. Wandering third-instar larvae were mounted on a slide between a sylgard cushion and a coverslip, which was held in place with orthodontic rubber bands. Live imaging was performed on a Quorum spinning disc confocal/ 1X8-1-Olympus inverted microscope equipped with a Hamamatsu 69100-13 camera and MetaMorph acquisition software. Time-lapse movies were taken at one frame per $2 \mathrm{~s}$ using a $40 \times$ air objective (NA 0.75). Analysis of microtubule dynamics was modified from Chen et al. (2012). Briefly, the number of EB1 comets was counted within $50 \mu \mathrm{m}$ dendritic segments beginning $10 \mu \mathrm{m}$ from the cell body of multidendritic sensory neurons. Quantification was based on three consecutive in-focus frames. RNAi against $C G 18278$ was used as a control. At least two dendritic segments were averaged for each cell, blinded to genotype. ImageJ software was used for image analysis.

Nerve crush assay and analysis. Larvae were positioned on their trachea with the ventral nerves closest to the top surface, and then segmental nerves were pinched tightly through the cuticle for $10 \mathrm{~s}$ with Dumostar number 5 forceps. Larvae were transferred to a grape plate with inactivated yeast and water mixture and kept alive for $7 \mathrm{~h}$ at $23^{\circ} \mathrm{C}$. Pinched larvae were then dissected and stained for the growth cone analysis, which was modified from Xiong et al. (2010). Stained crushed proximal stumps were analyzed for the presence of a growth cone while blinded to the genotype. To score a growth cone as sprouting, the axons must have at least five visible sprouts. Then, all analyzed crushed axons were combined for each genotype, and the fraction of sprouting stumps was generated. At least 12 larvae with a minimum of 55 nerves were analyzed for each genotype. $\chi^{2}$ test was performed to assess statistically significant differences among the genotypes.

\section{Results}

\section{Short stop is required for normal synaptic terminal growth at} the larval NMJ

To identify novel regulators of synaptic development, we conducted an anatomical screen on a subset of EMS mutants preselected for adult lethality or abnormalities in larval or adult locomotion. Third-instar larvae were dissected and stained with antibodies against the active zone component Bruchpilot (Wagh et al., 2006) and the essential glutamate receptor subunit DGluRIII (Marrus et al., 2004). We assessed various aspects of synaptic development, including the apposition of presynaptic active zones and postsynaptic glutamate receptor clusters, synaptic terminal overgrowth and undergrowth, and synaptic maintenance (Valakh et al., 2012). We focus here on a mutant that displayed dramatic NMJ overgrowth. The phenotype is apparent when visualizing terminal morphology by staining for the postsynaptic scaffolding protein Discs-large, the synaptic vesicle protein DVGLUT, and the presynaptic membrane as visualized by an antibody to HRP (Fig. 1A). Although the NMJ overgrowth is present at the synapse on every muscle, the number of synaptic boutons was quantified at the well-characterized muscle 4 type Ib NMJ. Mutant NMJs contain approximately twice as many boutons as in wild-type (WT) (Fig. 1A,C). To identify the gene responsible for the synaptic phenotype, we performed meiotic recombination and deficiency mapping and identified a small region in the second chromosome (50C6-50D2) that contains 34 genes. We then took a candidate approach, focusing on short stop (shot), a large gene in the region with a known function in neurons. The well-characterized null allele of shot, $\operatorname{shot}^{3}$ (Röper and Brown, 2003), failed to complement our new mutant, demonstrating that it is a new allele of shot. We name this new allele shot $t^{V V}$. Because $s h o t^{V V}$ is viable as a third-instar larvae, while all previously described alleles of shot are embryonic lethal, this new 
A
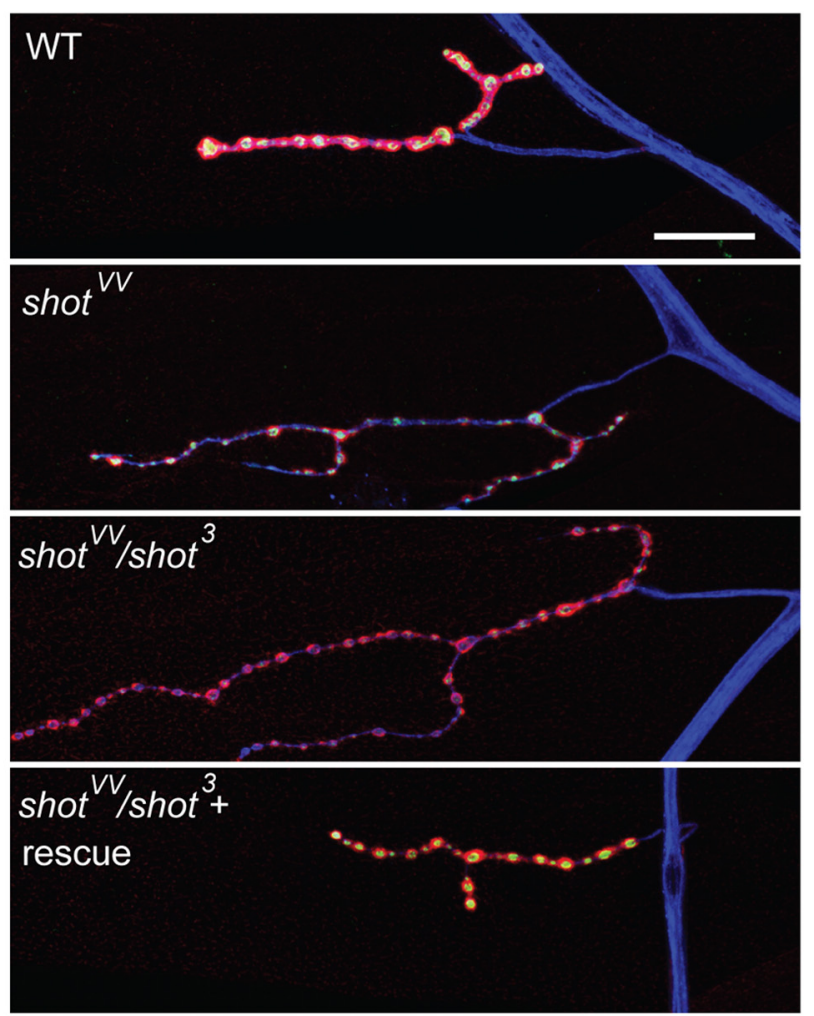

B
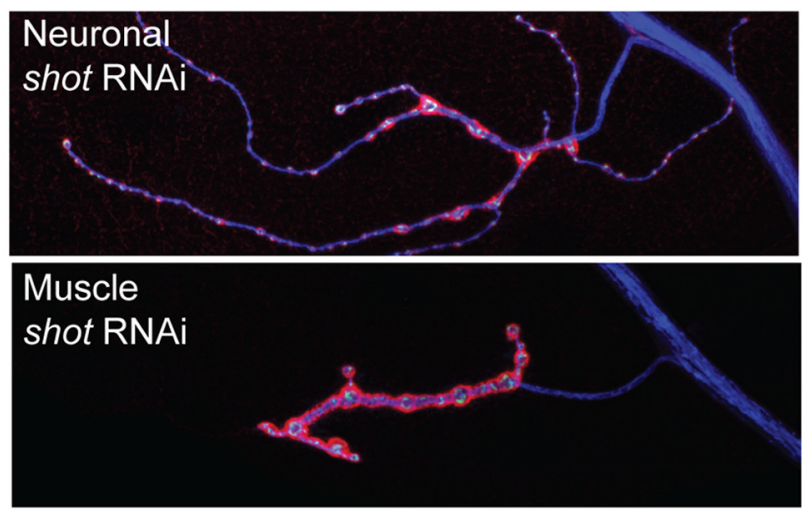

C

D

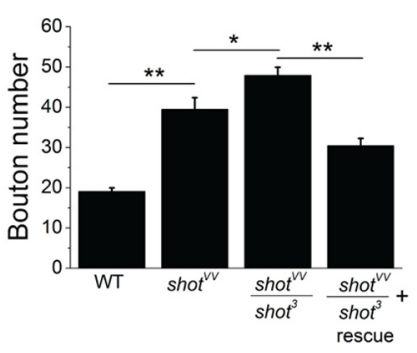

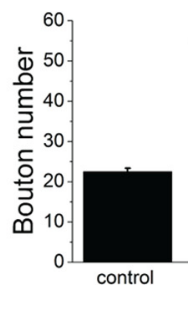

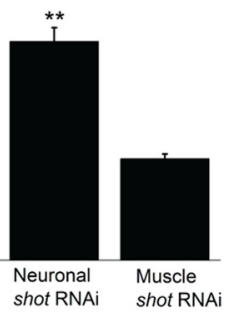

Figure 1. Synaptic terminal overgrowth at the NMJs in the shot mutant. A, Sample confocal images of muscle 4 type lb NMJs of WT, shot $t^{\nu /} /$ shot $^{\nu v}\left(\right.$ shot $\left.^{\nu \nu}\right)$, shot $t^{\nu v} /$ shot $^{3}$ and UAS-shot-FL-GFP/+ shot $^{\text {vv }} /$ shot $^{3}$; elav-Gal4/+ (shot $t^{v /} /$ shot $^{3}+$ rescue) stained for the postsynaptic DLG (red), presynaptic DVGLUT (green), and the membrane marker HRP (blue). Scale bar, $50 \mu \mathrm{m} . \boldsymbol{B}$, Sample confocal images of muscle 4 type Ib NMJs of UAS-dcr2, elav-Gal4/UAS-shot-RNAi (Neuronal shot RNAi), and UAS-dcr2; G7-Gal4/+; UAS-shot-RNAi/+ (Muscle shot RNAi). Muscle 4 type Ib bouton number of the genotypes listed in $\boldsymbol{A}$ are quantified in $\boldsymbol{C} .{ }^{* *} p<0.001$, compared with WT. ${ }^{*} p<0.05$, compared with WT. Phenotypes listed in $\boldsymbol{B}$ with addition of UAS-dcr2;; elav-Gal4/+ (control) were quantified in $\boldsymbol{D} .{ }^{* *} p<0.001$, compared with control. $n>15$ for each genotype. Error bars indicate SEM.

allele is likely a hypomorph. However, the NMJ overgrowth phenotype of $s h o t^{V V} /$ shot $^{3}$ is only slightly stronger than that of $\operatorname{shot} t^{V V}$ alone (Fig. $1 \mathrm{~A}, \mathrm{C}$ ), suggesting that the $\operatorname{shot}^{V V}$ allele is a relatively strong loss-of-function mutant, at least for its role in regulating NMJ terminal growth. We use the combination of $s h o t^{V V} /$ shot $^{3}$ for all subsequent experiments to avoid issues of second site mutations, and we will refer to this genotype as shot.

To test whether the synaptic overgrowth phenotype of shot is due to the absence of WT shot, we used the UAS/Gal4 system to express transgenic Shot in a tissue-specific manner. Expression of a full-length long isoform Shot-FL (Bottenberg et al., 2009) with the neuronal driver elav-Gal4 in the $s h o t^{V V} /$ shot $^{3}$ mutant background rescues the synaptic terminal overgrowth (Fig. $1 A, C$ ). In contrast, muscle expression of the same transgene with the strong muscle driver 24B-Gal4 was unable to rescue the phenotype. Hence, the NMJ overgrowth results from the loss of shot function and shot is required in neurons for normal synaptic development.

Because the nature of the mutation in $\operatorname{shot}^{V V}$ allele is unknown, we wanted to test whether loss of function of shot is sufficient to induce the overgrowth phenotype. Driving a transgenic RNAi targeting shot with the neuronal driver elav-Gal4 leads to an expansion of the NMJ that is very similar to that of the genetic mutant (Fig. 1B). Knockdown of shot in the muscle does not result in a synaptic phenotype (Fig. $1 B, D$ ). These data indicate that loss of function of shot in the nervous system is sufficient to cause synaptic overgrowth.
The Rod and Plakin domains are dispensable for the function of Shot in restraining NMJ growth

Spectraplakins are comprised of an N-terminal Calponin domain, a Plakin domain, a long rod domain with multiple Spectrin repeats, and a $\mathrm{C}$-terminal $\mathrm{EF}$ hand and mictrotubule-binding growth arrest-specific 2 (Gas2) domain (Röper et al., 2002). Spectraplakins perform different functions in different tissues, and domains that are crucial in one context may be dispensable in another (Bottenberg et al., 2009). To investigate the structural requirements of Shot for NMJ growth, we used a series of transgenes that expresses GFP-tagged shot mutants that were previously generated lacking individual domains (Lee and Kolodziej, 2002, Bottenberg et al., 2009); the structures of the transgenes are schematized in Figure $2 A$. For each deletion variant, we tested for transport of GFP-Shot out of the cell bodies and into the axons as well as genetic rescue of the shot NMJ overgrowth phenotype. We find that the full-length Shot, Shot-FL, is abundant in axons. We see similar axonal localization of Shot- $\Delta$ Calponin, which lacks one actin-binding Calponin homology domain, Shot- $\Delta$ Rod, lacking the large Spectrin repeat rod domain, and Shot- $\Delta$ Plakin, which lacks the large Plakin domain (Fig. 2B). However Shot$\Delta \mathrm{EF}$, in which the $\mathrm{Ca}^{2+}$ binding $\mathrm{EF}$ hand domain is deleted, and Shot- $\Delta$ Gas2, in which the microtubule-binding Gas2 domain is deleted, show very little axonal accumulation (Fig. $2 B$ ). Shot- $\Delta \mathrm{EF}$ shows strong expression in cell bodies (data not shown), suggesting that this calcium-binding EF hand domain may be required for efficient transport of Shot into the axon. 
A

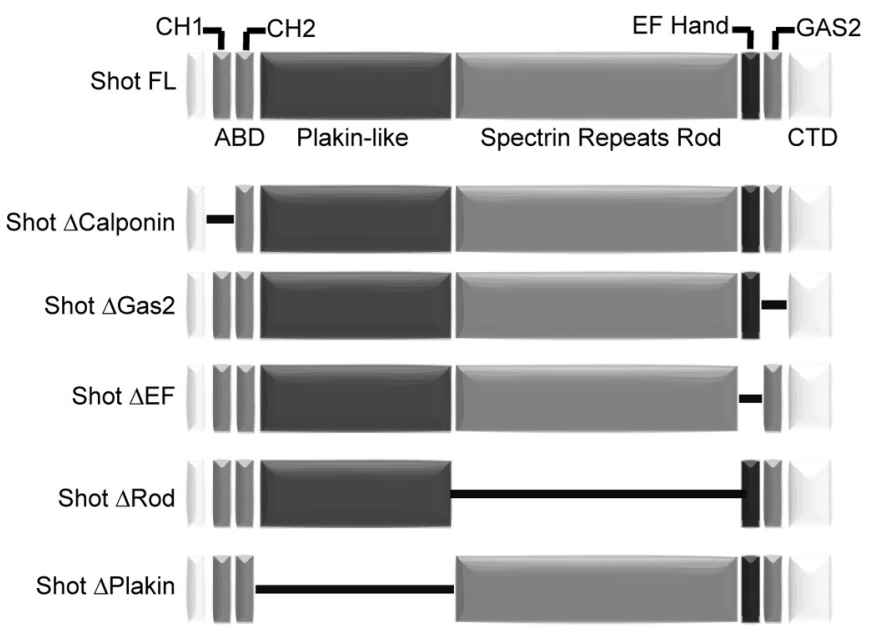

B

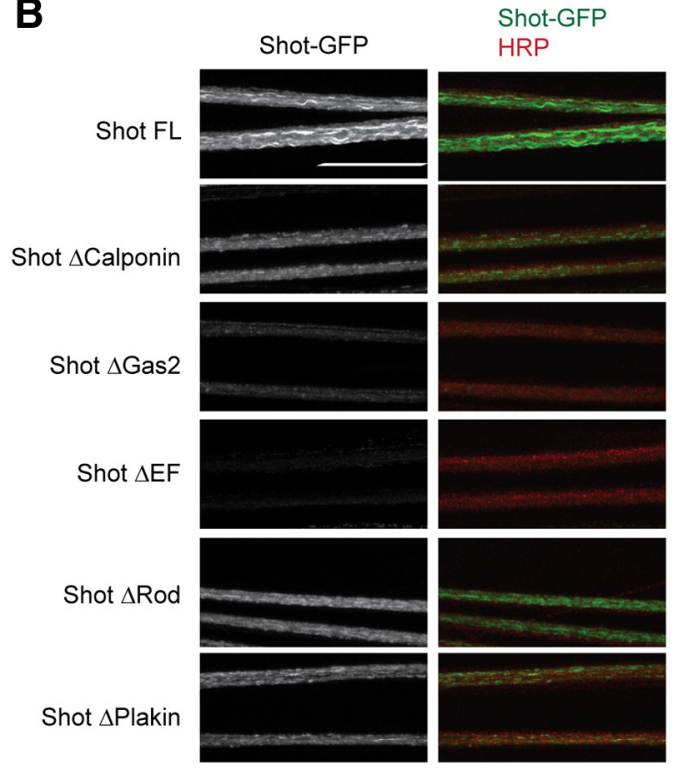

C

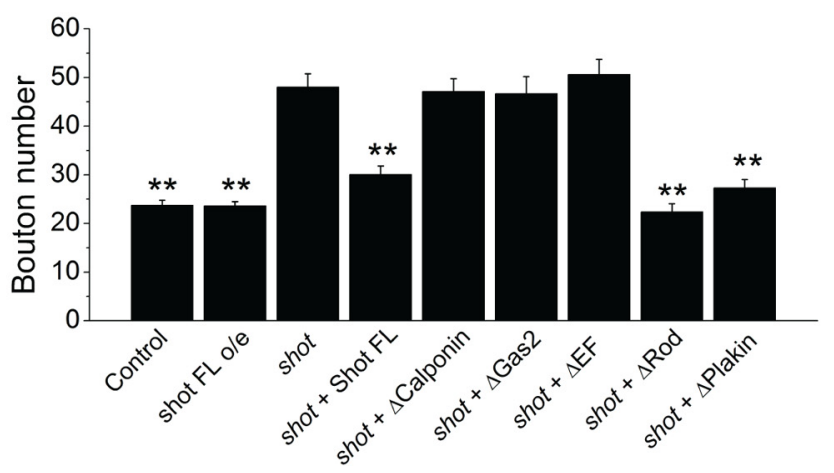

Figure 2. Rescue constructs missing the Rod domain and Plakin domain rescue the NMJ overgrowth phenotype of shot. A, Schematic representation of Short Stop and the deletion constructs. Shot $\Delta$ Calponin is a Shot $\mathrm{C}$ isoform, which lacks one of the two Calponin homology domains. The rest of the constructs were derived from Shot $\mathrm{A}$ isoform represented in $\boldsymbol{A}$ as Shot $\mathrm{FL}$ (full-length). All constructs are $\mathrm{COOH}$ GFP-tagged. $\boldsymbol{B}$, Sample confocal images of two nerves in the larvae expressing deletion constructs with the strong neuronal driver BG380-Gal 4 in the WT background. The nerves are stained for GFP (green) to label the expression of the constructs and HRP (red) to label the neuronal membrane. Scale bar, $50 \mu \mathrm{m}$. C, Quantification of the muscle 4 type Ib bouton number in

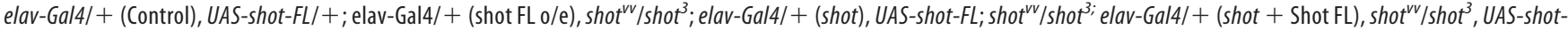
$\Delta$ Calponin; elav-Gal4l+ (shot $+\Delta$ Calponin), UAS-shot- $\Delta$ Gas $2 /+;$ shot $^{2 v} /$ shot $^{3} ;$ elav-Gal4l+ (shot $+\Delta$ Gas2), shot ${ }^{2 v} /$ shot $^{3}$, UAS-shot- $\Delta$ EFHand; elav-Gal4/ $+($ shot $+\Delta \mathrm{EF}) ;$

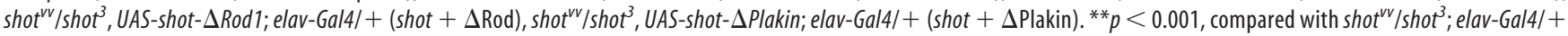
(shot). $n>15$ for each genotype. Error bars indicate SEM.

Having assessed the localization of these Shot deletion variants, we next tested whether expression of the transgene could rescue the NMJ overgrowth phenotype of the shot mutant. Expression of the full-length Shot-FL rescues the mutant, and overexpression of this transgene in a WT background does not affect NMJ growth (Figs. $1 A$ and $2 C$ ). We also observe robust rescue with Shot- $\Delta$ Plakin and Shot- $\Delta$ Rod (Fig. $2 C$ ). Hence, the large Plakin and rod domains, which are thought to serve as spacers between the actin and microtubule binding domains, are dispensable for the function of Shot in NMJ growth control. In contrast, expression of Shot- $\Delta$ Calponin is unable to rescue the mutant phenotype. Because this protein is present in axons at levels similar to that of the Shot- $\Delta$ Plakin and Shot- $\Delta$ Rod proteins, the failure to rescue likely indicates that the absence of the Calponin homology domain in this mutant abrogates its ability to restrain synaptic terminal growth. Finally, neither the Shot$\Delta \mathrm{EF}$ nor the Shot- $\Delta \mathrm{Gas} 2$ rescues the mutant phenotype (Fig. $2 C$ ). Hence, this formally demonstrates that those domains are required for function, but this may be the result of defects in axonal localization, protein function, or both. Together, this structure function analysis demonstrates that the various binding domains at the $\mathrm{N}$ - and C-terminus of Shot are important for its ability to restrain synaptic growth but that the spacing between those domains is not.

\section{Shot-dependent synaptic terminal overgrowth requires} Wallenda signaling

We wished to understand the molecular mechanism by which shot restrains NMJ growth during development. The overgrowth in shot is reminiscent of highwire mutants where absence of the 
A
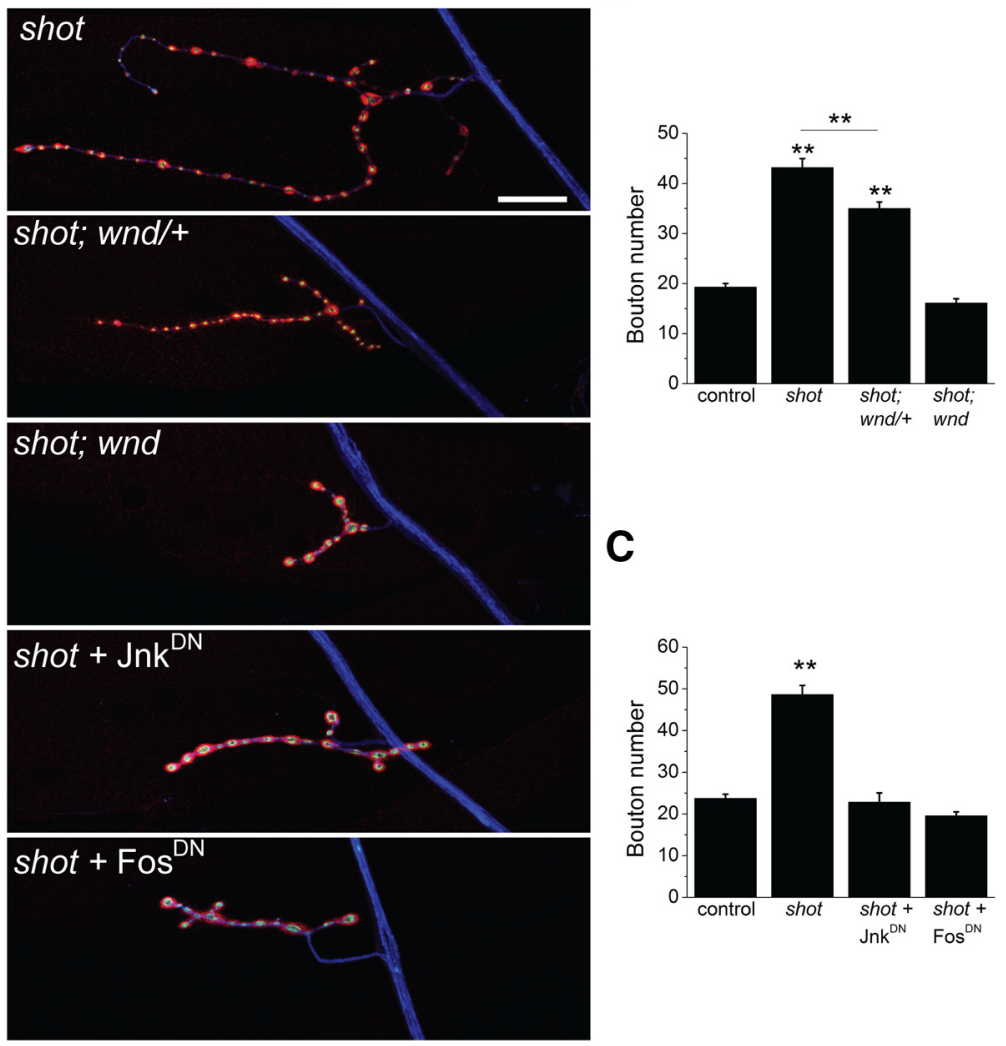

Figure 3. wallenda (DLK) mutation and its downstream factors suppress the NMJ overgrowth in shot. $\boldsymbol{A}$, Sample confocal

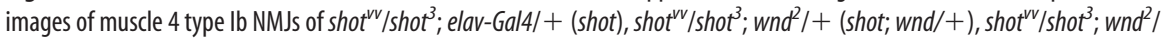
wnd $^{3}$ (shot; wnd), shot $t^{v v} /$ shot $^{3}$; elav-Gal4/UAS-bsk ${ }^{D N}\left(\right.$ shot $\left.+\mathrm{Jnk}^{\mathrm{DN}}\right)$, shot ${ }^{v v} /$ shot $^{3}$; elav-Gal4/UAS-fbz (shot + Fos $\left.{ }^{\mathrm{DN}}\right)$. B, Quantification of the muscle 4 type Ib bouton number in WT, shot ${ }^{v v} /$ shot $^{3}$ (shot), and shot ${ }^{v v} /$ shot $^{3}$; wnd $d^{2} /$ wnd ${ }^{3}$ (shot; wnd). C, Quantification of the muscle 4 type lb bouton number in elav-Gal4/+ (control) and the lower two genotypes in $\boldsymbol{A} .{ }^{* *} p<0.001$, compared with WT or control. $n>15$ for each genotype. Scale bar, $50 \mu \mathrm{m}$.

ubiquitin ligase Highwire leads to an increase in the levels of the MAPKKK Wallenda/DLK and subsequently increased downstream JNK/Fos signaling. Hence, we tested whether wallenda is necessary for the synaptic overgrowth phenotype of shot. Removing one copy of wallenda significantly suppresses the NMJ phenotype in shot mutants (Fig. $3 A, B$ ). Loss of both copies of wallenda in the shot mutant background $\left(\operatorname{shot}^{V V} /\right.$ shot $^{3}$; wnd $^{2}$ / $w n d^{3}$ ) results in full suppression (Fig. $3 A, B$ ). Hence, wallenda is required for shot-dependent synaptic terminal overgrowth, and the degree of overgrowth depends on the dose of Wallenda/DLK, suggesting that the extent of DLK activity is instructive for synaptic terminal overgrowth.

In the highwire mutant, wallenda-dependent NMJ overgrowth requires the downstream MAP kinase JNK as well as its target, the transcription factor Fos. To investigate the role of JNK and Fos for the shot phenotype, we used the same dominant-negative constructs used for the analysis of highwire (Collins et al., 2006). Expression of a dominant-negative transgene of either JNK or fos in neurons suppresses the increase in synaptic bouton number in the shot mutant (Fig. $3 A, C$ ). Hence, shot-dependent NMJ overgrowth requires the same signaling pathway as highwiredependent overgrowth, the MAP3K Wallenda/DLK, the MAPK JNK, and the transcription factor Fos.

\section{Shot does not regulate Wallenda protein levels}

In highwire mutants, absence of the ubiquitin ligase Highwire leads to an increase in the levels of Wallenda/DLK, which then activates the JNK/Fos pathway. Overexpression of Wallenda/DLK can also engage the JNK/Fos pathway to trigger NMJ overgrowth (Collins et al., 2006). Hence, increasing the levels of the Wallenda/DLK can overactivate this pathway, and this is the only known mechanism to activate Wallenda/DLK signaling in Drosophila. Because the shot phenotype also requires the Wallenda/DLK pathway, we wondered whether Shot also regulates the abundance of Wallenda/DLK. We used a previously generated and validated antibody (Collins et al., 2006) to assess the levels of Wallenda in whole-brain homogenates via Western blot. In WT larvae, Wallenda levels are very low; and as previously described, they are significantly increased in the highwire mutant. In contrast, in shot mutant larvae Wallenda protein levels are similar to those observed in WT (Fig. $4 A, B$ ). Similar results were obtained using immunohistochemistry: Wallenda protein is enriched in the synaptic neuropil of the highwire mutant but is at WT levels in the shot mutant (Fig. $4 C, D)$. Hence, although the Wallenda pathway is required for synaptic overgrowth in shot, the levels of Wallenda protein, unlike in highwire mutant, are not increased. Hence, shot does not function by controlling Wallenda levels.

\section{Shot inhibits Wallenda/DLK pathway activity}

The requirement of the Wallenda pathway for shot-dependent synaptic overgrowth is consistent with at least two models. First, the Wallenda pathway may play a permissive role in shot-dependent synaptic overgrowth. Here Wallenda would be necessary, but shot would function in an alternate pathway. Alternatively, Shot may act by inhibiting the Wallenda pathway activity. In this model, the Wallenda pathway would be upregulated in the shot mutant. To distinguish between these two possibilities, we probed JNK pathway activity in the shot mutant. JNK pathway activity can be assayed in Drosophila via a LacZ enhancer trap that is inserted into the JNK phosphatase puckered (Martín-Blanco et al., 1998), which is a transcriptional target of the JNK pathway. puc-LacZ is expressed at low levels in larval neurons from WT flies and is elevated when the Wallenda/DLK pathway is overactivated in highwire mutants (Xiong et al., 2010). We quantified the levels of puc-LacZ expression in neurons from both WT and shot mutant larvae. The level of puc-LacZ expression is increased approximately threefold over WT in shot (Fig. $5 A, B)$, demonstrating that the JNK pathway is upregulated in the shot mutant.

Because the JNK pathway can be upregulated by a variety of upstream kinases (Davis, 2000), we tested whether Wallenda is necessary for activation of the JNK pathway. As previously described (Xiong et al., 2010), we were unable to generate recombinants between wallenda and puc-LacZ. Instead, we tested the role of Wallenda/DLK using a previously verified RNAi line (Xiong et al., 2010). Knockdown of shot expression using RNAi recapitulates the NMJ overgrowth phenotype (Fig. 1B) and also 
A

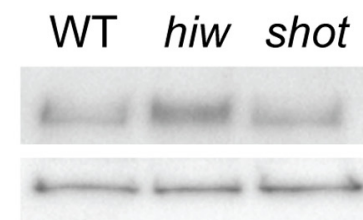

C
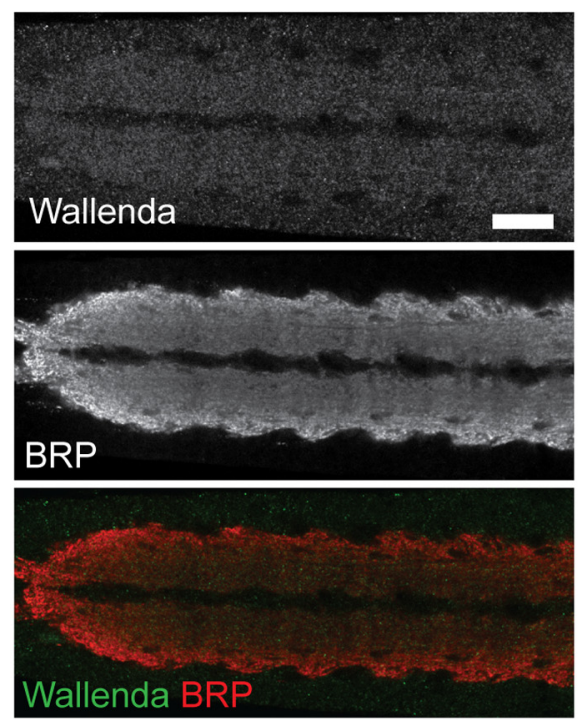

B
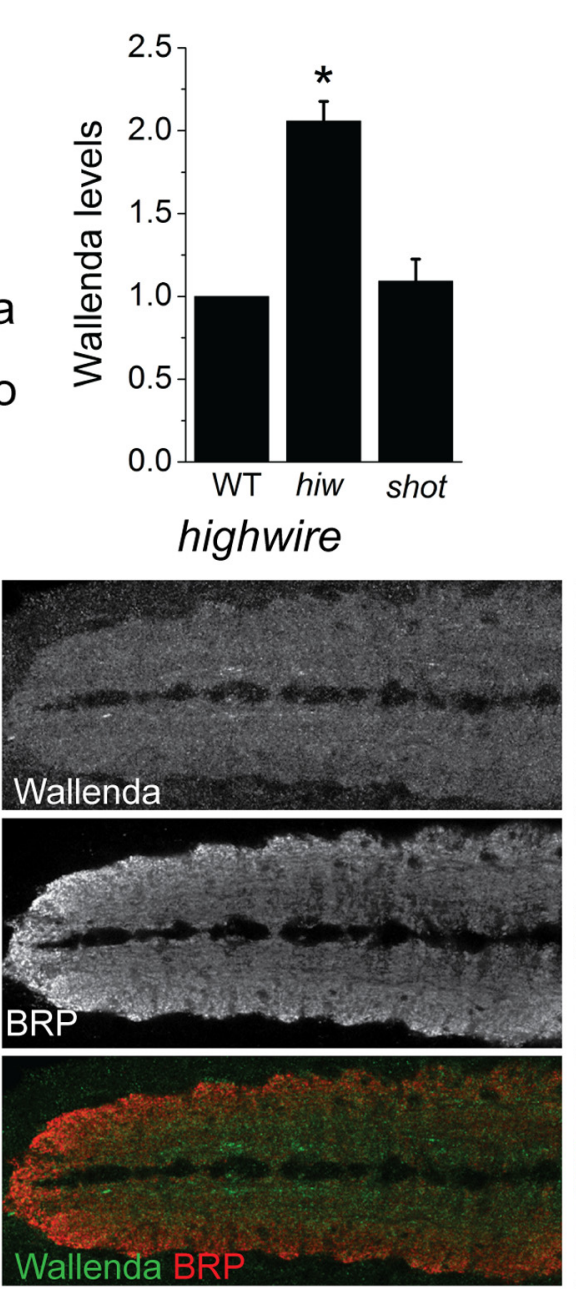

Western Blot
Wallenda

Armadillo
D Immunihistochemistry
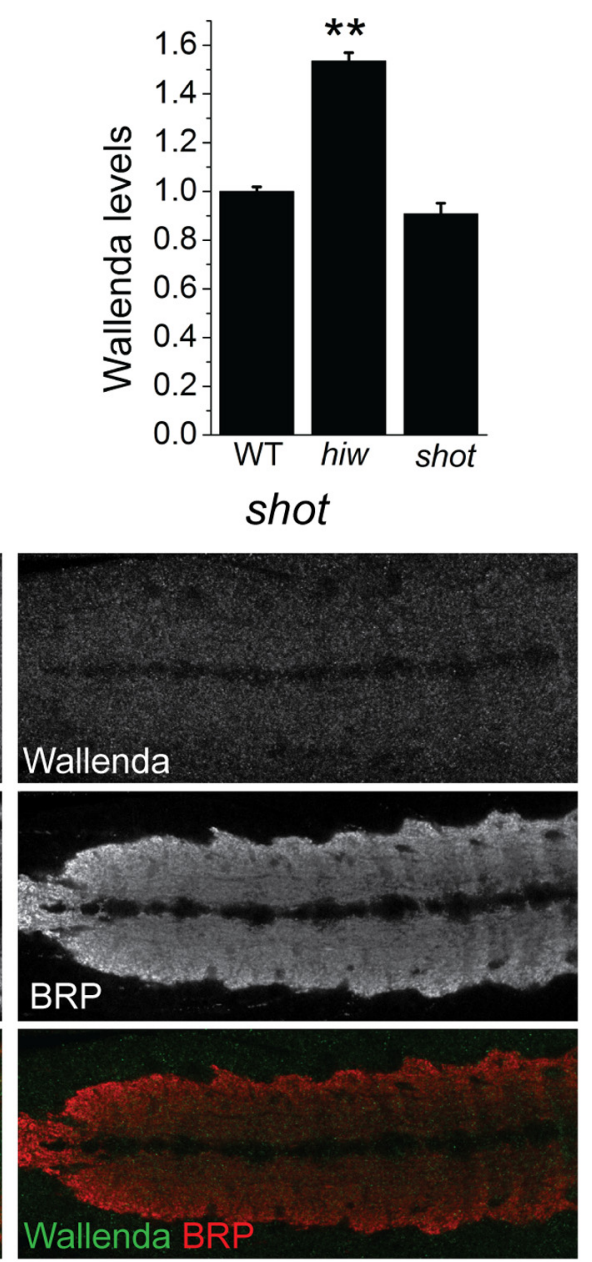

Figure 4. Wallenda/DLK levels are not increased in shot mutant. $A$, Representative Western blots of extracts from larval brain and ventral nerve cords of WT, highwire $\Delta C$ mutant (hiw), and $\operatorname{shot}^{\nu v /} /$ shot $^{3}$ (shot) probed for Wallenda and Armadillo as a loading control. B. The levels of the protein are quantified from three independent Western blots and then normalized to control from each blot. ${ }^{*} p<0.01$, compared with WT. C, Staining for endogenous Wallenda in the ventral nerve cords of third-instar larvae for WT, highwire $\Delta\left(\right.$ mutant (highwire), and shot ${ }^{t v} /$ shot ${ }^{3}$ (shot). The Wallenda antibody is labeled in green, and the neuropil is labeled with synaptic marker BRP (red). The Wallenda intensity in the neuropil was measured for each genotype and normalized to WT levels. $\boldsymbol{D}$, The quantification is presented as mean \pm SEM. ${ }^{* *} p<0.001$, compared with WT. $n>9$ brains for each genotype. Scale bar, $50 \mu \mathrm{m}$.

leads to increased expression of puc-lacZ as in the classical mutant (Fig. 5). Double knockdown of shot and wallenda suppresses this increase in puc-lacZ expression (Fig. $5 \mathrm{~A}, \mathrm{C}$ ). The suppression of $p u c$-lacZ expression with wallenda RNAi is not the result of dilution of Gal4 activity in the presence of multiple UAS lines because we failed to find suppression with two control UASRNAi constructs (shot RNAi, $3.0 \pm 0.1$ a.u.; shot RNAi + CG13772 RNAi, $3.2 \pm 0.1$ a.u; shot RNAi + CG18278 RNAi, $3.6 \pm 0.2$ a.u.). Together, these data demonstrate that in the shot mutant the Wallenda/DLK signaling pathway is upregulated; and so when Shot function is unperturbed, the Wallenda/DLK pathway is inhibited. Because Shot does not function by controlling the levels of Wallenda as Highwire does, Shot must function via a novel mechanism to control the activation of Wallenda/DLK.

\section{Cytoskeletal disruption and the activation of the Wallenda/DLK pathway}

Short Stop acts as an actin-microtubule cross-linker; and in shot null embryos, the microtubule skeleton is less stable (Alves-Silva et al., 2012). To explore the possibility that disrupting the cyto- skeleton might activate Wallenda/DLK, we first tested whether the inhibition of shot in larvae also destabilizes the cytoskeleton. We assessed in vivo microtubule dynamics by expressing the microtubule plus end binding-protein EB1-GFP in sensory neurons with $p p k$-Gal4. EB1-GFP labels the growing ends of microtubules in structures called "comets," the number of which reflect the number of growing microtubules (Chen et al., 2012). Knockdown of short stop results in an approximate doubling of comets labeled with EB1 (control RNAi: $2.7 \pm 1.5$ comets, $n=21$ neurons; shot RNAi: $5.3 \pm 0.8$ comets, $n=14$ neurons; $p=0.001$ ), demonstrating that the microtubule network is more dynamicl less stable (Fig. 6A). The role of short stop in stabilizing the cytoskeleton and in the activation of the Wallenda/DLK pathway in shot mutants is consistent with microtubule destabilization being the mechanism that activates Wallenda/DLK.

If cytoskeletal destabilization activates Wallenda/DLK activation, then other mutants that disrupt the cytoskeleton would be predicted to activate Wallenda/DLK. To this end, we targeted two subunits of the T-complex protein-1 (TCP1) complex. TCP1 is a cytosolic chaperone for tubulin and actin. Data from yeast and 
A
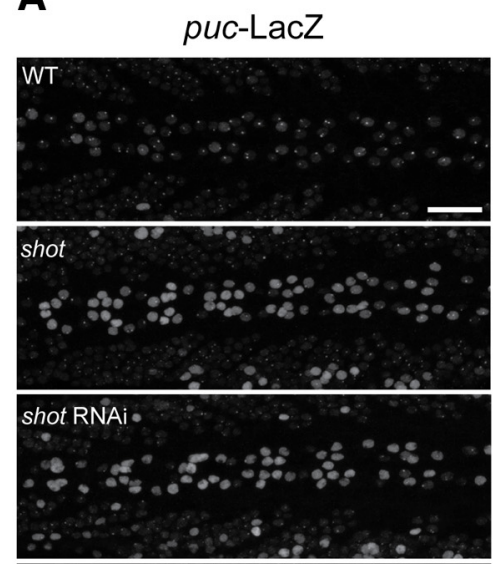

shot RNAi;

whd RNAi
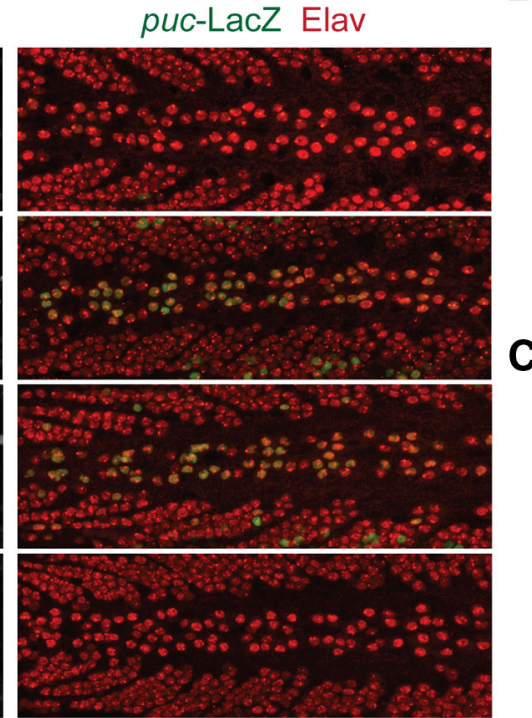

B
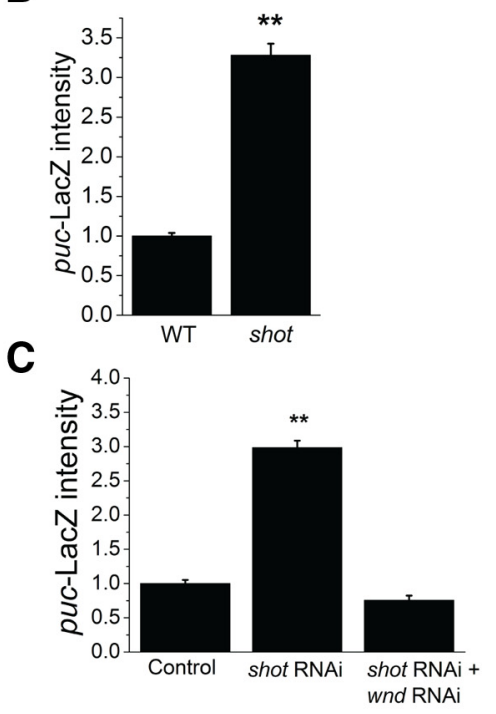

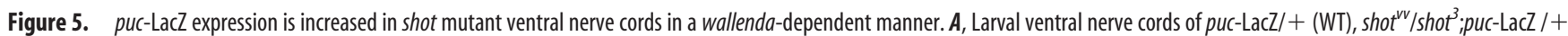
(shot), elav-Gal4,UAS-dcr2/+;UAS-shot-RNAi/puc-LacZ (shot RNAi), and elav-Gal4,UAS-dcr2/UAS-wnd-RNAi; UAS-shot-RNAi/puc-LacZ (shot RNAi; wnd RNAi) stained for puc-LacZ expression in green (left) and Elav protein (red). puc-LacZ expression is weak in the WT ventral nerve cords but is increased in shot mutant and shot RNAi larvae. The levels of puc-LacZ are quantified in the neurons along the dorsal midline identified by Elav protein expression of the genotypes from $\boldsymbol{A}$ in addition to elav-Gal4,UAS-dcr2/+;puC-LaCZ/+(Control) are represented in $\boldsymbol{B}$ and $\mathbf{C}$ as mean \pm SEM. ${ }^{* *} p<$ 0.001, compared with WT or control (elav-Gal4, UAS-dcr2I+). $n>9$ brains for each genotype. Scale bar, $50 \mu \mathrm{m}$.

mammalian cell lines show that, when this complex is disrupted, the microtubule network is destabilized (Ursic et al., 1994; Grantham et al., 2006). We find that RNAi-mediated downregulation of the genes for either TCP $1 \alpha$ or TCP $1 \beta$, two required subunits of the TCP1 complex, also activates the JNK pathway, and this activation requires wallenda (Fig. 6B). Hence, this independent manipulation recapitulates our findings with short stop and is consistent with the model that the activation of the DLK/ Wallenda pathway in shot is the result of destabilization of the cytoskeleton.

\section{Shot mutants accelerate the regenerative response to injury via the Wallenda/DLK pathway}

In addition to its role in synapse development, Wallenda/DLK signaling also promotes axonal regeneration after injury in worms, flies, and mammals (Hammarlund et al., 2009; Yan et al., 2009; Xiong et al., 2010; Shin et al., 2012). In Drosophila, loss of highwire leads to an increase in the levels of Wallenda/DLK that speeds the regenerative response (Xiong et al., 2010). We wondered whether it would be possible to promote regenerative sprouting by activating the Wallenda/DLK pathway without an increase in Wallenda levels. shot mutants have normal levels of Wallenda protein but increased pathway activity, so we tested whether this activation is sufficient to hasten the regenerative response to injury. We labeled two axons per nerve using $\mathrm{m} 12$ Gal4 to drive expression of a membrane-tagged GFP, crushed peripheral nerves as previously described (Xiong et al., 2010), and assessed the regenerative potential of axons in Drosophila larvae. To quantify the response, we counted the fraction of injured nerves that show a large growth cone $7 \mathrm{~h}$ after crush (for details of blinded analysis, see Materials and Methods). Seven hours after injury, WT axons are just beginning to regenerate, with only $15 \%$ showing the regenerative response of a sprouting growth cone. On the other hand, in shot mutants $52 \%$ of axons have new axonal sprouts (Fig. 7 $A, B$ ). We next investigated whether the improved regenerative response in shot requires wallenda. RNAi knockdown of shot shows a very similar increase in the number of regenerative sprouts as the classical shot mutant. However, when combined with wallenda RNAi the enhanced regenerative response is eliminated (Fig. 7C,D). These data demonstrate that the Wallenda/DLK pathway is required for more rapid formation of regenerative axonal branches in the absence of shot. Hence, loss of shot improves the regenerative response to injury by activating the Wallenda/DLK pathway.

\section{Discussion}

The MAPKKK Wallenda/DLK is an important regulator of synaptic terminal development and the axonal response to injury (Collins et al., 2006; Hammarlund et al., 2009; Xiong et al., 2010; Shin et al., 2012). However, the mechanisms that activate this kinase are not well understood. Here we show that mutation of the spectraplakin Short stop leads to activation of Wallenda/DLK signaling, resulting in synaptic terminal overgrowth and an accelerated regenerative response to axonal injury.

\section{A new function for the spectraplakin short stop}

Spectraplakins are huge, multidomain proteins that bind to both actin and microtubules to regulate cytoskeletal dynamics. The family of spectraplakins consists of mammalian bpag1/dystonin (Sawamura et al., 1991) and ACF7/MAC1 (Leung et al., 1999), Drosophila short stop (shot)/kakapo (Gregory and Brown, 1998), and C. elegans vab-10 (Suozzi et al., 2012). Spectraplakins function in many cellular processes, including regulating ER-Golgi transport in mammalian sensory neurons (Ryan et al., 2012a, $2012 \mathrm{~b}$ ) and mediating polarized locomotion of skin stem cells upon injury (Wu et al., 2011). They also play an essential role in axons, as mutations in mammalian spectraplakins lead to peripheral neuropathy in both mice and humans (Bernier et al., 1995; Edvardson et al., 2012). In Drosophila, the spectraplakin short stop has been extensively studied for its role in embryonic axon outgrowth and its regulation of microtubule dynamics (Lee et al., 2000; Sanchez-Soriano et al., 2009; Alves-Silva et al., 2012). All 
A
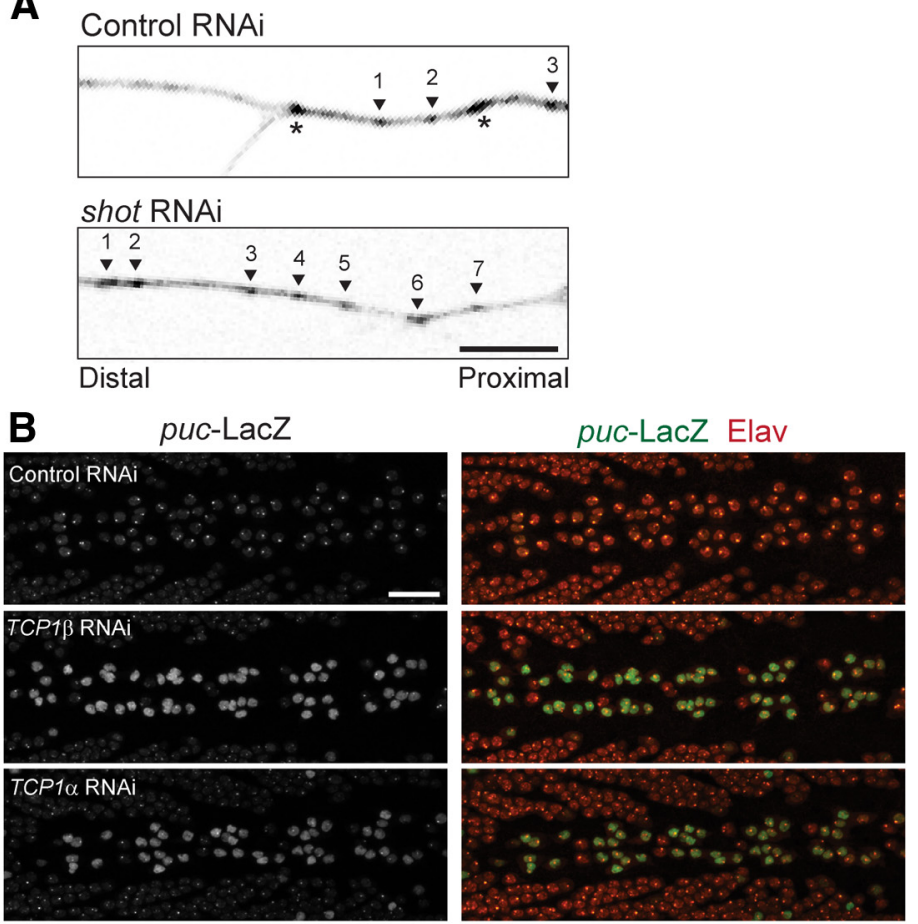

C
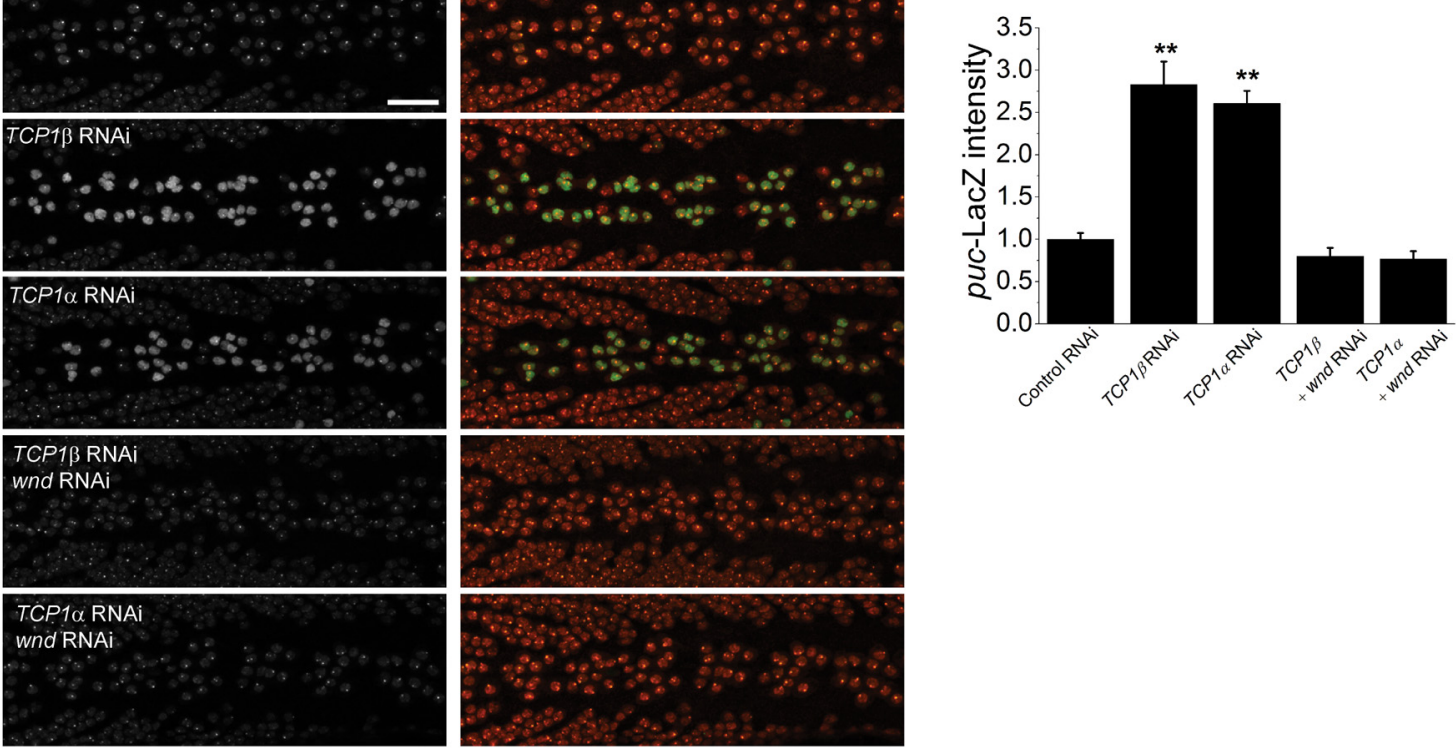

Figure 6. Cytoskeletal disruption and the activation of the Wallenda/DLK pathway. $A$, Growing microtubule plus-ends marked by EB1-GFP expression driven by $p p k$-Gal4 in a branch of a sensory neuron in UAS-dcr2/UAS-CG18287-RNAi; ppk-Gal4, UAS-EB1-GFP (Control RNAi) and UAS-dcr2/+;UAS-shot-RNAi/ppk-Gal4, UAS-EB1-GFP (shot RNAi) larvae. Arrowheads indicate moving EB1-GFP comets, and asterisks indicate stationary puncta. Only the moving comets were counted in the area. Knockdown of shot expression results in approximate doubling of the growing microtubule plus-ends compared with control. Scale bar, $10 \mu \mathrm{m}$. B, Larval ventral nerve cords of elav-Gal4,UAS-dcr2/+;puc-LacZ/+ (Control), elav-Gal4, UAS-dcr2/+;UAS-TCP1 $\beta$-RNAi/puC-LaCZ (TCP1 $\beta$ RNAi), elav-Gal4, UAS-dcr2/+;UAS-TCP1 $\alpha$-RNAi/puc-LacZ (TCP1 $\alpha$-RNAi), elav-Gal4, UAS-dcr2/UAS-wnd-RNAi; UAS-TCP1 $\beta$-RNAi/puc-LacZ (TCP1 $\beta$-RNAi; wnd RNAi), elav-Gal4, UAS-dcr2/UAS-wndRNAi; UAS-TCP1 $\alpha$-RNAi/puC-LacZ (TCP1 $\alpha$-RNAi; wnd RNAi), stained for puc-LacZ expression in green (left) and Elav protein (red). puc-LacZ expression is increased in TCP1 RNAi larvae. C, The levels of $p$ UC-LaCZ are quantified in the neurons along the dorsal midline identified by Elav protein expression are represented as mean \pm SEM. ${ }^{* *} p<0.001$, compared with WT. $n>9$ brains for each genotype. Scale bar, $50 \mu \mathrm{m}$.

prior loss-of-function mutants in shot are embryonic lethal, and these strong alleles have severe impairments of their cytoskeleton and poor axon outgrowth, leading axons to "stop short" of their targets (Lee et al., 2000). This embryonic phenotype is in apparent contradiction to the larval phenotype we describe for the shot ${ }^{V V}$ allele, in which the synaptic terminal is overgrown with additional synaptic boutons. However the $s h o t^{V V}$ allele is a hypomorph and motor axons successfully navigate to their targets, so this mutant must retain sufficient function to allow for axonal outgrowth. Although shot ${ }^{V V}$ behaves as a hypomorph, the molecular lesion was not identified, and so it is plausible that this allele is a neomorph and that its regulation of DLK may not reflect the normal function of the protein. However, we find that RNAi knockdown of shot generates the same phentoypes as $s h t^{V V}$ and leads to the same activation of Wallenda/DLK. Hence, both the shot ${ }^{V V}$ allele and shot RNAi provide evidence for a new function for shot, namely, as a negative regulator of Wallenda/DLK signaling. It will be interesting to determine whether mammalian spectraplakins also restrain DLK signaling and whether dysregulated MAP kinase pathways may mediate some of the phenotypes of spectraplakin mutants, such as peripheral neuropathy.

\section{Activation of Wallenda/DLK}

A series of recent studies highlights the central role of DLK in the developing and injured mammalian nervous system. DLK is required for normal developmental cell death in motor and sensory neurons (Ghosh et al., 2011; Itoh et al., 2011), for Wallerian degeneration of injured peripheral axons (Miller et al., 2009), for cell death and axon degeneration of retinal ganglion cells in models of glaucoma (Watkins et al., 2013, Welsbie et al., 2013), and for the proregenerative preconditioning response in injured DRG axons (Shin et al., 2012). Because DLK appears to be central to the neuronal injury response, there is great interest in understanding its mechanism of activation. In particular, it is important to understand how axon injury leads to the activation of DLK.

To date, methods that increase the levels of DLK are the best understood mechanism for increasing DLK activity. In worms, flies, and mice, loss of the PHR ubiquitin ligase leads to an increase in the levels of DLK (Nakata et al., 2005; Collins et al., 2006; Babetto et al., 2013); and in worms and flies, there are extensive data demonstrating that this activates the kinase. Similarly, in both worms and flies, the overexpression of DLK is sufficient to 
A
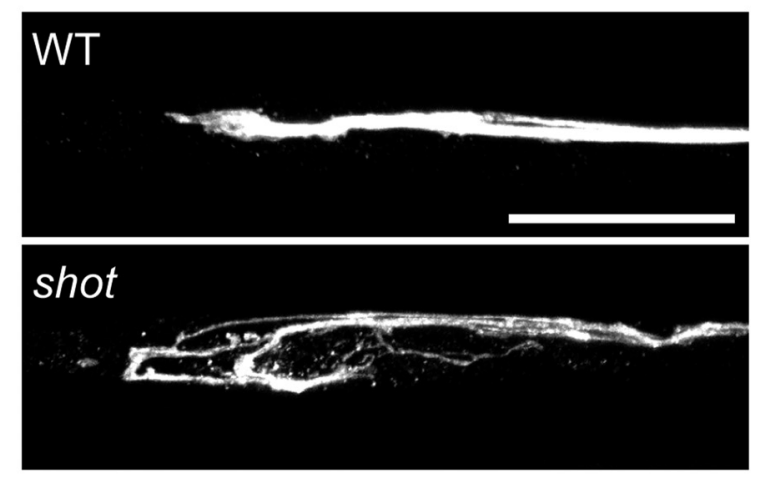

C
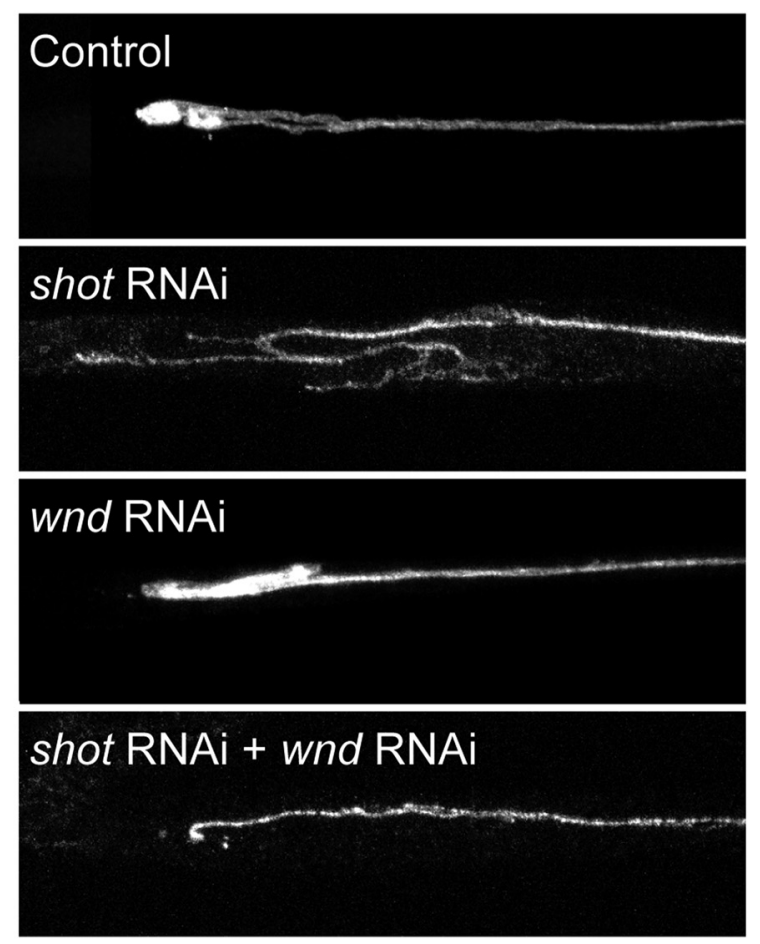

B

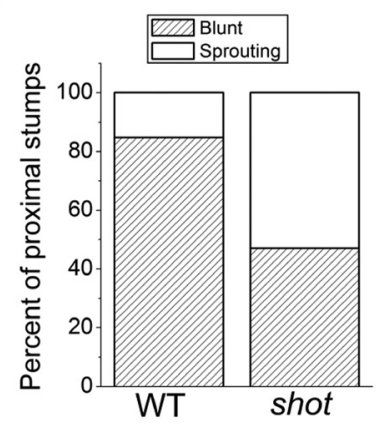

D

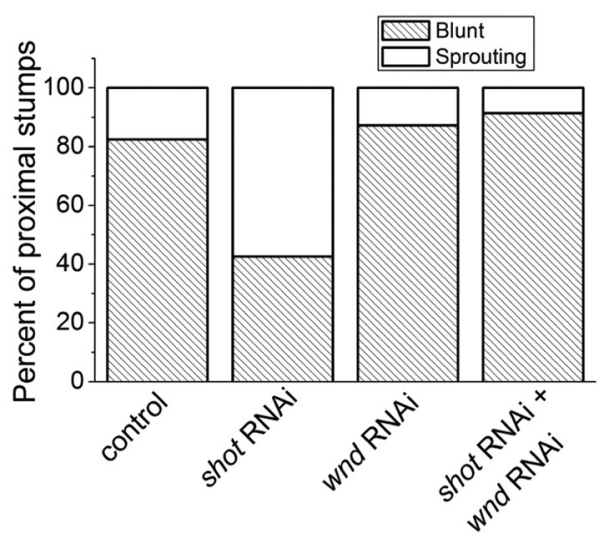

Figure 7. shot mutants have enhanced axonal sprouting after injury. Axons are labeled by driving expression of UAS-mCD8-GFP by $m 12-G a l 4$. Axons from (A) WT animals do not yet form extensive new branches by $7 \mathrm{~h}$ after injury, whereas in $\operatorname{shot}^{\nu v} / \mathrm{shot}^{3}$ (shot) mutants a large growth cone with multiple sprouting sites can be seen more frequently than in WT $\left(p<0.001, \chi^{2}\right.$ test, $n>30$ axons for each genotype). The number of blunt versus sprouting axonal stumps was counted, and their frequencies are presented in $B$. C, Same analysis was done on UAS-dcr2;; UAS-mCD8-GFP, m12-Gal4/+ (control), UAS-dcr2; UAS-mCD8-GFP, m12-Gal4/UAS-shot-RNAi; (shot RNAi), UAS-dcr2; UAS-wnd-RNAil+; UAS-mCD8-GFP, m12-Gal4/+ (wnd RNAi), and UAS-dcr2; UAS-wnd-RNAi IUAS-mCD8-GFP,m12-Gal4; UAS-shot-RNAi/ + (shot RNAi; wnd RNAi) and quantified in D. $n>50$ axons for each genotype. Scale bar, $25 \mu \mathrm{m}$.

activate the DLK signaling pathway. In Drosophila, injury leads to a loss of the PHR ubiquitin ligase Highwire (Xiong et al., 2010), potentially via autophagosomal degradation (Shen and Ganetzky, 2009; Tian et al., 2011), which in turn leads to an increase in DLK and may be a method of injury-induced activation. In mammals, a positive feedback loop between DLK and JNK inhibits Phr1-dependent degradation of DLK, increasing the levels of DLK and activating the pathway (Huntwork-Rodriguez et al., 2013). In addition, a calciumdependent activation mechanism was recently demonstrated in $C$. elegans (Yan and Jin, 2012), which is very exciting because calcium influx is an early step after axon injury. However, the key hexapeptide sequence that mediates this calcium-dependent regulation is absent from both Drosophila and mouse DLK, suggesting that additional activation mechanisms for DLK may exist. Indeed, our data show that loss of the spectraplakin shot can also activate DLK and leads to the hypothesis that cytoskeletal disruptions may activate DLK. Our findings demonstrate that loss of function of shot leads to activation of Wallenda/DLK without a concomitant increase in the levels of Wallenda/DLK. Hence, loss of shot is not acting upstream or in concert with Highwire because loss of Highwire or components of the Highwire ubiquitin ligase complex lead to increased levels of Wallenda/DLK (Collins et al., 2006; Wu et al., 2007). The shot mutant is, to our knowledge, the first manipulation that activates Wallenda/DLK signaling in Drosophila without altering the levels of Wallenda/DLK. Hence, loss of shot must activate Wallenda/DLK via a novel mechanism. We hypothesize that this mechanism is related to the biochemical function of Shot, which is to stabilize the cytoskeleton by simultaneously binding both actin and microtubules. Prior studies demonstrate that shot null mutants have a destabilized microtubule network (Alves-Silva et al., 2012), and we demonstrate 
that the microtubule network is more dynamic in shot RNAi knockdown larvae. We propose that a destabilized cytoskeleton activates Wallenda/DLK. Consistent with this model, we show that mutations in either of two subunits of the TCP-1 complex, which like Shot regulates both the actin and microtubule cytoskeleton, leads to activation of DLK signaling. By demonstrating this activation of DLK signaling, our results support and extend the work of Bounoutas et al. (2011) who demonstrated that microtubule disruption leads to DLK-dependent changes in protein levels in C. elegans. Moreover, our findings are consistent with studies in mammalian cell culture demonstrating that drugs that destabilize microtubules can activate MAP kinase signaling (Wang et al., 1998; Stone and Chambers, 2000; Yang et al., 2007), as well as studies in Drosophila showing that genetic disruption of the cytoskeleton can activate JNK signaling (Massaro et al., 2009).

Axonal injury as a result of trauma or neurotoxic insults, such as chemotherapy drug treatment, is accompanied by a change in microtubule network stability. We propose a model in which DLK functions as a sensor of microtubule network stability. When the cytoskeleton is destabilized as a result of injury, DLK will be activated. The consequence of that activation will depend on downstream signaling pathways and may differ by cellular compartment. For example, DLK in the distal axon will promote axonal degeneration (Miller et al., 2009), whereas DLK activation in proximal axons will facilitate the retrograde transport of injury signals that can activate regenerative and/or apoptotic gene expression programs (Shin et al., 2012; Watkins et al., 2013).

\section{Promoting regeneration by activating DLK}

In the mammalian PNS, DLK is required for the preconditioning response that boosts the efficacy of peripheral DRG axon regeneration after a prior nerve injury (Shin et al., 2012). In both worms and flies, activation of DLK by increasing its abundance improves the regenerative response in the absence of a prior nerve injury (Hammarlund et al., 2009; Xiong et al., 2010). Hence, it is attractive to speculate that activation of DLK in the absence of injury may also improve regeneration in mammalian axons. Our findings with shot suggest that relatively mild disruptions to the axonal cytoskeleton can activate DLK and accelerate the regenerative response in Drosophila in the absence of a prior trauma. Future studies will test whether pharmacological agents that disrupt the cytoskeleton can activate DLK in mammalian neurons and whether such activation promotes axon regeneration.

In conclusion, our study demonstrates that, in the absence of Shot, Wallenda/DLK signaling is activated resulting in synaptic terminal overgrowth and more rapid regenerative axonal sprouting. The role of Shot as an actin-microtubule cross-linker suggests that Wallenda/DLK is activated by cytoskeletal disruption and suggests novel approaches for controlling DLK activity in the injured or diseased nervous system.

\section{References}

Alves-Silva J, Sánchez-Soriano N, Beaven R, Klein M, Parkin J, Millard TH, Bellen HJ, Venken KJ, Ballestrem C, Kammerer RA, Prokop A (2012) Spectraplakins promote microtubule-mediated axonal growth by functioning as structural microtubule-associated proteins and EB1-dependent +TIPs (tip interacting proteins). J Neurosci 32:9143-9158. CrossRef Medline

Babetto E, Beirowski B, Russler EV, Milbrandt J, DiAntonio A (2013) The Phr1 ubiquitin ligase promotes injury-induced axon self-destruction. Cell Rep 3:1422-1429. CrossRef Medline

Bernier G, Brown A, Dalpé G, De Repentigny Y, Mathieu M, Kothary R (1995) Dystonin expression in the developing nervous system predominates in the neurons that degenerate in dystonia musculorum mutant mice. Mol Cell Neurosci 6:509-520. CrossRef Medline

Bloom AJ, Miller BR, Sanes JR, DiAntonio A (2007) The requirement for Phr1 in CNS axon tract formation reveals the corticostriatal boundary as a choice point for cortical axons. Genes Dev 21:2593-2606. CrossRef Medline

Bottenberg W, Sanchez-Soriano N, Alves-Silva J, Hahn I, Mende M, Prokop A (2009) Context-specific requirements of functional domains of the Spectraplakin Short stop in vivo. Mech Dev 126:489-502. CrossRef Medline

Bounoutas A, Kratz J, Emtage L, Ma C, Nguyen KC, Chalfie M (2011) Microtubule depolymerization in Caenorhabditis elegans touch receptor neurons reduces gene expression through a p38 MAPK pathway. Proc Natl Acad Sci U S A 108:3982-3987. CrossRef Medline

Brand AH, Perrimon N (1993) Targeted gene expression as a means of altering cell fates and generating dominant phenotypes. Development 118: 401-415. Medline

Chen L, Stone MC, Tao J, Rolls MM (2012) Axon injury and stress trigger a microtubule-based neuroprotective pathway. Proc Natl Acad Sci U S A 109:11842-11847. CrossRef Medline

Collins CA, Wairkar YP, Johnson SL, DiAntonio A (2006) Highwire restrains synaptic growth by attenuating a MAP kinase signal. Neuron 51: 57-69. CrossRef Medline

Culican SM, Bloom AJ, Weiner JA, DiAntonio A (2009) Phr1 regulates retinogeniculate targeting independent of activity and ephrin-A signalling. Mol Cell Neurosci 41:304-312. CrossRef Medline

Daniels RW, Collins CA, Gelfand MV, Dant J, Brooks ES, Krantz DE, DiAntonio A (2004) Increased expression of the Drosophila vesicular glutamate transporter leads to excess glutamate release and a compensatory decrease in quantal content. J Neurosci 24:10466-10474. CrossRef Medline

Davis RJ (2000) Signal transduction by the JNK group of MAP kinases. Cell 103:239-252. CrossRef Medline

DiAntonio A, Haghighi AP, Portman SL, Lee JD, Amaranto AM, Goodman CS (2001) Ubiquitination-dependent mechanisms regulate synaptic growth and function. Nature 412:449-452. CrossRef Medline

Dietzl G, Chen D, Schnorrer F, Su KC, Barinova Y, Fellner M, Gasser B, Kinsey K, Oppel S, Scheiblauer S, Couto A, Marra V, Keleman K, Dickson BJ (2007) A genome-wide transgenic RNAi library for conditional gene inactivation in Drosophila. Nature 448:151-156. CrossRef Medline

Edvardson S, Cinnamon Y, Jalas C, Shaag A, Maayan C, Axelrod FB, Elpeleg O (2012) Hereditary sensory autonomic neuropathy caused by a mutation in dystonin. Ann Neurol 71:569-572. CrossRef Medline

Eresh S, Riese J, Jackson DB, Bohmann D, Bienz M (1997) A CREB-binding site as a target for decapentaplegic signalling during Drosophila endoderm induction. EMBO J 16:2014-2022. CrossRef Medline

Ghosh AS, Wang B, Pozniak CD, Chen M, Watts RJ, Lewcock JW (2011) DLK induces developmental neuronal degeneration via selective regulation of proapoptotic JNK activity. J Cell Biol 194:751-764. CrossRef Medline

Grantham J, Brackley KI, Willison KR (2006) Substantial CCT activity is required for cell cycle progression and cytoskeletal organization in mammalian cells. Exp Cell Res 312:2309-2324. CrossRef Medline

Gregory SL, Brown NH (1998) kakapo, a gene required for adhesion between and within cell layers in Drosophila, encodes a large cytoskeletal linker protein related to plectin and dystrophin. J Cell Biol 143:12711282. CrossRef Medline

Hammarlund M, Nix P, Hauth L, Jorgensen EM, Bastiani M (2009) Axon regeneration requires a conserved MAP kinase pathway. Science 323:802806. CrossRef Medline

Huntwork-Rodriguez S, Wang B, Watkins T, Ghosh AS, Pozniak CD, Bustos D, Newton K, Kirkpatrick DS, Lewcock JW (2013) JNK-mediated phosphorylation of DLK suppresses its ubiquitination to promote neuronal apoptosis. J Cell Biol 202:747-763. CrossRef Medline

Itoh A, Horiuchi M, Wakayama K, Xu J, Bannerman P, Pleasure D, Itoh T (2011) ZPK/DLK, a mitogen-activated protein kinase kinase kinase, is a critical mediator of programmed cell death of motoneurons. J Neurosci 31:7223-7228. CrossRef Medline

Klapper R (2000) The longitudinal visceral musculature of Drosophila melanogaster persists through metamorphosis. Mech Dev 95:47-54. CrossRef Medline

Kolodziej PA, Jan LY, Jan YN (1995) Mutations that affect the length, fasciculation, or ventral orientation of specific sensory axons in the Drosophila embryo. Neuron 15:273-286. CrossRef Medline

Lee S, Kolodziej PA (2002) Short Stop provides an essential link between F-actin and microtubules during axon extension. Development 129: 1195-1204. Medline

Lee S, Harris KL, Whitington PM, Kolodziej PA (2000) short stop is allelic to 
kakapo, and encodes rod-like cytoskeletal-associated proteins required for axon extension. J Neurosci 20:1096-1108. Medline

Leung CL, Sun D, Zheng M, Knowles DR, Liem RK (1999) Microtubule actin cross-linking factor (MACF): a hybrid of dystonin and dystrophin that can interact with the actin and microtubule cytoskeletons. J Cell Biol 147:1275-1286. CrossRef Medline

Lewcock JW, Genoud N, Lettieri K, Pfaff SL (2007) The ubiquitin ligase Phrl regulates axon outgrowth through modulation of microtubule dynamics. Neuron 56:604-620. CrossRef Medline

Marrus SB, Portman SL, Allen MJ, Moffat KG, DiAntonio A (2004) Differential localization of glutamate receptor subunits at the Drosophila neuromuscular junction. J Neurosci 24:1406-1415. CrossRef Medline

Martín-Blanco E, Gampel A, Ring J, Virdee K, Kirov N, Tolkovsky AM, Martinez-Arias A (1998) puckered encodes a phosphatase that mediates a feedback loop regulating JNK activity during dorsal closure in Drosophila. Genes Dev 12:557-570. CrossRef Medline

Massaro CM, Pielage J, Davis GW (2009) Molecular mechanisms that enhance synapse stability despite persistent disruption of the spectrin/ankyrin/microtubule cytoskeleton. J Cell Biol 187:101-117. CrossRef Medline

Miller BR, Press C, Daniels RW, Sasaki Y, Milbrandt J, DiAntonio A (2009) A dual leucine kinase-dependent axon self-destruction program promotes Wallerian degeneration. Nat Neurosci 12:387-389. CrossRef Medline

Nakata K, Abrams B, Grill B, Goncharov A, Huang X, Chisholm AD, Jin Y (2005) Regulation of a DLK-1 and p38 MAP kinase pathway by the ubiquitin ligase RPM-1 is required for presynaptic development. Cell 120: 407-420. CrossRef Medline

Nix P, Hisamoto N, Matsumoto K, Bastiani M (2011) Axon regeneration requires coordinate activation of $\mathrm{p} 38$ and JNK MAPK pathways. Proc Natl Acad Sci U S A 108:10738-10743. CrossRef Medline

Röper K, Brown NH (2003) Maintaining epithelial integrity: a function for gigantic spectraplakin isoforms in adherens junctions. J Cell Biol 162: 1305-1315. CrossRef Medline

Röper K, Gregory SL, Brown NH (2002) The "spectraplakins": cytoskeletal giants with characteristics of both spectrin and plakin families. J Cell Sci 115:4215-4225. CrossRef Medline

Ryan SD, Bhanot K, Ferrier A, De Repentigny Y, Chu A, Blais A, Kothary R (2012a) Microtubule stability, Golgi organization, and transport flux require dystonin-a2-MAP1B interaction. J Cell Biol 196:727-742. CrossRef Medline

Ryan SD, Ferrier A, Kothary R (2012b) A novel role for the cytoskeletal linker protein dystonin in the maintenance of microtubule stability and the regulation of ER-Golgi transport. Bioarchitecture 2:2-5. CrossRef Medline

Sanchez-Soriano N, Travis M, Dajas-Bailador F, Gonçalves-Pimentel C, Whitmarsh AJ, Prokop A (2009) Mouse ACF7 and drosophila short stop modulate filopodia formation and microtubule organisation during neuronal growth. J Cell Sci 122:2534-2542. CrossRef Medline

Sanyal S (2009) Genomic mapping and expression patterns of C380, OK6 and D42 enhancer trap lines in the larval nervous system of Drosophila. Gene Expr Patterns 9:371-380. CrossRef Medline

Sawamura D, Li K, Chu ML, Uitto J (1991) Human bullous pemphigoid antigen (BPAG1): amino acid sequences deduced from cloned cDNAs predict biologically important peptide segments and protein domains. J Biol Chem 266:17784-17790. Medline

Schaefer AM, Hadwiger GD, Nonet ML (2000) rpm-1, a conserved neuronal gene that regulates targeting and synaptogenesis in C. elegans. Neuron 26:345-356. CrossRef Medline

Shen W, Ganetzky B (2009) Autophagy promotes synapse development in Drosophila. J Cell Biol 187:71-79. CrossRef Medline

Shin JE, Cho Y, Beirowski B, Milbrandt J, Cavalli V, DiAntonio A (2012) Dual leucine zipper kinase is required for retrograde injury signaling and axonal regeneration. Neuron 74:1015-1022. CrossRef Medline

Stone AA, Chambers TC (2000) Microtubule inhibitors elicit differential effects on MAP kinase (JNK, ERK, and p38) signaling pathways in human KB-3 carcinoma cells. Exp Cell Res 254:110-119. CrossRef Medline

Suozzi KC, Wu X, Fuchs E (2012) Spectraplakins: master orchestrators of cytoskeletal dynamics. J Cell Biol 197:465-475. CrossRef Medline

Tian X, Li J, Valakh V, DiAntonio A, Wu C (2011) Drosophila Rael controls the abundance of the ubiquitin ligase Highwire in post-mitotic neurons. Nat Neurosci 14:1267-1275. CrossRef Medline

Tian X, Wu C (2013) The role of ubiquitin-mediated pathways in regulating synaptic development, axonal degeneration and regeneration: insights from fly and worm. J Physiol 591:3133-3143. CrossRef Medline

Ursic D, Sedbrook JC, Himmel KL, Culbertson MR (1994) The essential yeast Tcpl protein affects actin and microtubules. Mol Biol Cell 5:10651080. CrossRef Medline

Valakh V, Naylor SA, Berns DS, DiAntonio A (2012) A large-scale RNAi screen identifies functional classes of genes shaping synaptic development and maintenance. Dev Biol 366:163-171. CrossRef Medline

Wagh DA, Rasse TM, Asan E, Hofbauer A, Schwenkert I, Dürrbeck H, Buchner S, Dabauvalle MC, Schmidt M, Qin G, Wichmann C, Kittel R, Sigrist SJ, Buchner E (2006) Bruchpilot, a protein with homology to ELKS/ CAST, is required for structural integrity and function of synaptic active zones in Drosophila. Neuron 49:833-844. CrossRef Medline

Wan HI, DiAntonio A, Fetter RD, Bergstrom K, Strauss R, Goodman CS (2000) Highwire regulates synaptic growth in Drosophila. Neuron 26: 313-329. CrossRef Medline

Wang TH, Wang HS, Ichijo H, Giannakakou P, Foster JS, Fojo T, Wimalasena J (1998) Microtubule-interfering agents activate c-Jun N-terminal kinase/ stress-activated protein kinase through both Ras and apoptosis signalregulating kinase pathways. J Biol Chem 273:4928-4936. CrossRef Medline

Watkins TA, Wang B, Huntwork-Rodriguez S, Yang J, Jiang Z, EasthamAnderson J, Modrusan Z, Kaminker JS, Tessier-Lavigne M, Lewcock JW (2013) DLK initiates a transcriptional program that couples apoptotic and regenerative responses to axonal injury. Proc Natl Acad Sci U S A 110:4039-4044. CrossRef Medline

Weber U, Paricio N, Mlodzik M (2000) Jun mediates Frizzled-induced $\mathrm{R} 3 / \mathrm{R} 4$ cell fate distinction and planar polarity determination in the Drosophila eye. Development 127:3619-3629. Medline

Welsbie DS, Yang Z, Ge Y, Mitchell KL, Zhou X, Martin SE, Berlinicke CA, Hackler L Jr, Fuller J, Fu J, Cao LH, Han B, Auld D, Xue T, Hirai S, Germain L, Simard-Bisson C, Blouin R, Nguyen JV, Davis CH, et al. (2013) Functional genomic screening identifies dual leucine zipper kinase as a key mediator of retinal ganglion cell death. Proc Natl Acad Sci U S A 110:4045-4050. CrossRef Medline

Wu C, Wairkar YP, Collins CA, DiAntonio A (2005) Highwire function at the Drosophila neuromuscular junction: spatial, structural, and temporal requirements. J Neurosci 25:9557-9566. CrossRef Medline

Wu C, Daniels RW, DiAntonio A (2007) DFsn collaborates with Highwire to down-regulate the Wallenda/DLK kinase and restrain synaptic terminal growth. Neural Dev 2:16. CrossRef Medline

Wu X, Shen QT, Oristian DS, Lu CP, Zheng Q, Wang HW, Fuchs E (2011) Skin stem cells orchestrate directional migration by regulating microtubule-ACF7 connections through GSK3 $\beta$. Cell 144:341-352. CrossRef Medline

Xiong X, Collins CA (2012) A conditioning lesion protects axons from degeneration via the Wallenda/DLK MAP kinase signaling cascade. J Neurosci 32:610-615. CrossRef Medline

Xiong X, Wang X, Ewanek R, Bhat P, DiAntonio A, Collins CA (2010) Protein turnover of the Wallenda/DLK kinase regulates a retrograde response to axonal injury. J Cell Biol 191:211-223. CrossRef Medline

Xiong X, Hao Y, Sun K, Li J, Li X, Mishra B, Soppina P, Wu C, Hume RI, Collins CA (2012) The Highwire ubiquitin ligase promotes axonal degeneration by tuning levels of Nmnat protein. PLoS Biol 10:e1001440. CrossRef Medline

Yan D, Jin Y (2012) Regulation of DLK-1 kinase activity by calciummediated dissociation from an inhibitory isoform. Neuron 76:534-548. CrossRef Medline

Yan D, Wu Z, Chisholm AD, Jin Y (2009) The DLK-1 kinase promotes mRNA stability and local translation in C. elegans synapses and axon regeneration. Cell 138:1005-1018. CrossRef Medline

Yang Y, Zhu X, Chen Y, Wang X, Chen R (2007) p38 and JNK MAPK, but not ERK1/2 MAPK, play important role in colchicine-induced cortical neurons apoptosis. Eur J Pharmacol 576:26-33. CrossRef Medline

Yao KM, White K (1994) Neural specificity of elav expression: defining a Drosophila promoter for directing expression to the nervous system. J Neurochem 63:41-51. CrossRef Medline

Zhen M, Huang X, Bamber B, Jin Y (2000) Regulation of presynaptic terminal organization by C. elegans RPM-1, a putative guanine nucleotide exchanger with a RING-H2 finger domain. Neuron 26:331-343. CrossRef Medline 